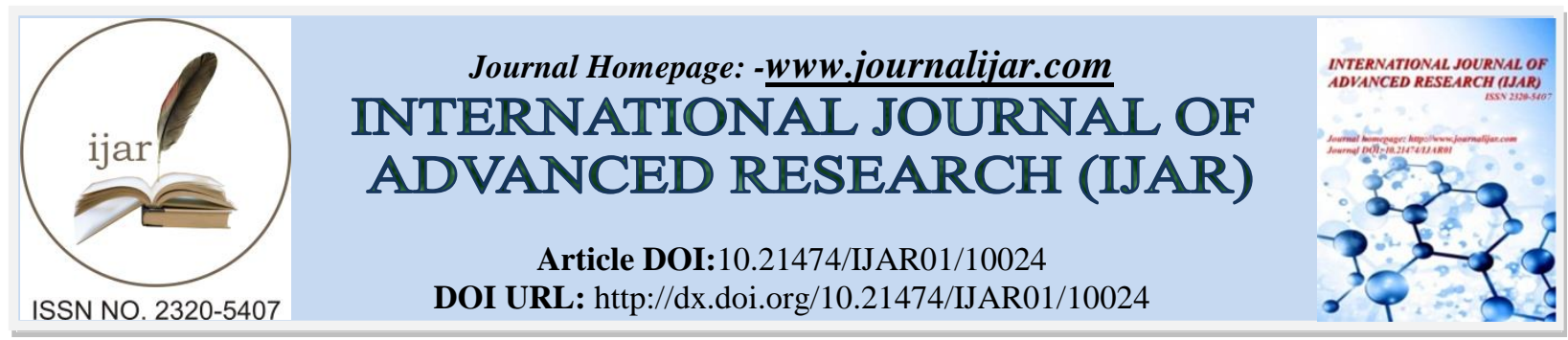

RESEARCH ARTICLE

\title{
DETERMINATION OF HG IN HAIR OF MINERS IN ABU HAMMED CITY (INDISCRIMINATE MINING AREA) USING DIRECT MERCURY ANALYZER, SUDAN.
}

\author{
M.Ismail ${ }^{1}$, A. S. Elhag ${ }^{2}$ and Elamin.E.Elamin ${ }^{1}$ \\ 1. Chemistry Department ,Faculty of Science, University of Khartoum. \\ 2. Sudan Atomic Energy Commission.
}

\section{Manuscript Info}

Manuscript History

Received: 10 September 2019

Final Accepted: 12 October 2019

Published: November 2019

Key words:-

Mercury, hair, direct mercury analyzer.

\begin{abstract}
The aim of this study was to evaluate the total mercury in hair samples of miners in Abu hammed (indiscriminate mining area) city using Direct Mercury Analyzer (DMA). Thirty samples were collected from different locations. Locations are divided depending on which type of work was done. Direct Mercury Analyzer (DMA) was used to determine the mercury in hair samples. The results show that the mean elemental concentration of $\mathrm{Hg}$ were 9.4 and $24 \mathrm{ppb}$ in location one and location 2, respectively and standard division of value of 15 and $21 \mathrm{ppb}$ in location one and location 2, respectively.
\end{abstract}

Copy Right, IJAR, 2019,. All rights reserved.

\section{Introduction:-}

Mercury is a heavy metal of known toxicity, it exists in several forms such an inorganic mercury, which includes metallic mercury and mercury vapor $\left(\mathrm{Hg}^{0}\right)$ and mercurous $\left(\mathrm{Hg} 2^{++}\right)$or mercuric $\left(\mathrm{Hg}^{++}\right)$salts; and organic mercury, which includes compounds in which mercury is bonded to a structure containing carbon atoms (methyl, ethyl, phenyl, or similar groups). The biological behavior, pharmacokinetics, and clinical significance of the various forms of mercury vary with chemical structure ${ }^{(1)}$.

Mercury $(\mathrm{Hg})$ is a dangerous metal and its presence in the environment and in the human food chain is a matter of increasing concern. It is a toxic element that has not physiologic function in human body, but exposure to $\mathrm{Hg}$ can rise a wide range of effects, e.g., inattention, memory disturbance, learning problems, impairment of social behavior, and low intelligence quotient. Although high or repeated exposure to different forms of $\mathrm{Hg}$ can have serious health consequences, the most important toxicity risk that $\mathrm{Hg}$ poses to humans is as methylmercury ( $\mathrm{MeHg}$ ) which exposure is mainly through consumption of fish. Generally, more than the $80 \%$ of the $\mathrm{Hg}$ in hair is as $\mathrm{MeHg}$, which is taken up by the hair follicles as $\mathrm{MeHg}$-cysteine complexes. Considering that hair grows ca. $1 \mathrm{~cm}$ per month and that the half-life averages of $\mathrm{MeHg}$ in hair is about 65 days (range of about 35100 days), it would be possible to quantify the $\mathrm{Hg}$ load due to a long period of exposure ${ }^{(2)}$.

The presence of $\mathrm{Hg}$ in hair is generally evaluated by different techniques such as the cold vapor atomic absorption spectroscopy (CV-AAS), inductively coupled plasma atomic emission spectrometry (ICP-AES) and Direct Mercury Analyzer (DMA). All these techniques are sensitive, precise and supply reliable and reproducible data, but, on the other hand, they are very time-consuming techniques due to the pretreatment of samples ${ }^{(2)}$.

Corresponding Author:-M.Ismail.

Address:- Chemistry Department ,Faculty of Science ,University of Khartoum. 
Mercury and its compounds occur naturally in the environment, but the use of them in industry and their release into the atmosphere by the burning of fossil fuels and the processing of ores has increased environmental levels. The world consumption of mercury has been decreasing due to public awareness of its toxicity. However, the amount of mercury already emitted and being recycled in the environment and new emissions from natural and anthropogenic sources is considerable. This increased concern about the health of persons exposed to very low environmental mercury concentrations is because mercury causes sub-clinical effects at low concentrations. The symptoms are difficult to detect and measure. For example, slightly increased levels of mercury in hair have been associated with decreases in academic ability. Also, reduced productivity and development of asthenic vegetive syndrome, a subtle behavior change, can occur $^{(3)}$.

Because at present there is no indicator to monitor the total environment to assess changes in man's exposure to mercury, a method for such environmental monitoring is required. It has been reported that mercury accumulates in the following matrices, any one of which could be used as an indicator of environmental mercury levels: lake sediments, bird feathers, food, fish, surface seawater, air and scalp hair ${ }^{(3)}$.

However, the availability of samples for any type of monitoring is important. In this case, of all the matrices mentioned, the easiest to collect, transport and store is human scalp hair. Mercury has been measured in human hair in forensic studies, for dietary reasons, in toxic and health care work and to examine environmental concentrations in polluted and unpolluted regions. ${ }^{(3)}$.

Mercury $(\mathrm{Hg})$ is considered as a global pollutant, because $\mathrm{Hg}^{0}$ is the predominant form of atmospheric $\mathrm{Hg}$, which has a long residence time in the atmosphere from 0.5 to 2 years. It can be transported and deposited to remote places even $1000 \mathrm{~km}$ away from the sources. Furthermore, $\mathrm{Hg}$ can be converted to methyl mercury (Me- $\mathrm{Hg}$ ) and accumulated in the food chain, posing a potential threat to humans' health. The concern of $\mathrm{Hg}$ pollution arises from the health effects caused by $\mathrm{Me}-\mathrm{Hg}$ through the consumption of fish and marine products ${ }^{(4)}$.

$\mathrm{Hg}$ is released to atmosphere from natural and anthropogenic sources. Coal combustion, waste incineration, metal mining, refining and manufacturing and chlorine-alkali production are currently major anthropogenic source categories in the industrialized world. Human activities emit both elemental $\mathrm{Hg}\left(\mathrm{Hg}^{0}\right)$ with a long life in the atmosphere and reactive gaseous mercury (RGM) and particulate $\mathrm{Hg}$, which are short lived in the air and deposited near the emission source. Mercury is also released into the atmosphere by a number of natural processes, including volcanoes and geothermal activities, evasion from surficial soils, water bodies, vegetation surfaces, wild fires, as well as the re-emission of deposited mercury. Mercury released from natural sources is believed to be mainly $\mathrm{Hg}^{0(4)}$. The main sources of mercury can be classified as: atmospheric deposition, erosion sources, urban sources, and mining: which is the important source for mercury ${ }^{(5)}$.

Analyses of hair samples enable to precisely evaluate the mineral concentration inside the body. Therefore, hair works as a dosimeter and the detected quantity is proportional to the concentration of mercury in the organism ${ }^{(6)}$. It also allows the evaluation of the levels of pollution through time. It is possible to tell how much mercury a person has been exposed to by testing their hair, blood and urine. According to the World Health Organization (3) (WHO2000)mercury and its compounds have been classified as one of most hazardous substances for living organisms. This is above all connected with the emission of mercury into the environment, the processes of redistribution, and the influence of mercury on human health ${ }^{(7)}$.

Mercury may be introduced into the human body by different ways: the respiratory tract (mercury vapor), the gastrointestinal tract (alkyl- and inorganic compounds) and also through the skin (vapor, organic compounds). In the case of occupational exposure, it is assumed that the main route of introduction for this metal into the organism is inhalation ( $80 \%$ of mercury is absorbed in this way). In the process of inhalation, elemental mercury vapor is transported to the systemic circulation of blood, where it is partially catalytically oxidized. An important role in the metabolism of mercury is played which form complexes with mercury. Inorganic compounds of mercury accumulate mainly in the liver and kidneys. Some organic compounds (phenyl-mercury) metabolize in the human body into inorganic compounds. Methylmercury collects in the central nervous system (and is very dangerous for the fetus $)^{(6)}$.

The aim of the study was to evaluate the total mercury in hair samples of miners in Abu hammed (indiscriminate mining area) city using Direct Mercury Analyzer (DMA). 


\section{Materials and Methods:-}

\section{Sampling and samples preparation}

A participants were randomly selected using the lottery method with replacement at the time of data collection and consented to the study, after the purpose of the study have been explained to them. Thirty hair samples were collected from indiscriminate mining of miners, samples length varies between 3 and $5 \mathrm{~cm} ; 0.5$ and $2.0 \mathrm{~g}$, stored in polyethylene bags prior to analysis.

\section{Preparation of standards}

Mercury stock standard solution (1000ppm) was prepared. Mercury working standards were prepared from the stock solution (1000ppm) on a daily basis and checked for stability of absorption before taking the readings. Mercury working standards make successive dilutions of the stock mercury solution to obtain standards containing $100 \mathrm{ppm}$ and $10 \mathrm{ppm}$ as shown Table $1.50 \mu \mathrm{g} / \mathrm{Kg}$ (50ppb) working standard, take $1 \mathrm{~mL}$ of the $5 \mathrm{ppm}$ Stock solution and dilute up to $100 \mathrm{~mL}$ were prepared. $150 \mu \mathrm{g} / \mathrm{Kg}(500 \mathrm{ppb})$ working standard, take $5 \mathrm{~mL}$ of the $10 \mathrm{ppm}$ solution and dilute up to $100 \mathrm{~mL}$ were prepared. $\mathrm{Hcl}$ (Con) with high purity grade or equivalent was used for standard preparation. Also reagent water will be used, high purity oxygen and mercury free will be used to avoid contamination.

Table 1:-Preparation of Calibration Standards of DMA-80

\begin{tabular}{|c|c|c|c|c|}
\hline NO. & $\begin{array}{l}\text { Conc. of the } \\
\text { stock }\end{array}$ & $\begin{array}{l}\text { Volume } \\
\text { taken } \mathrm{ml}\end{array}$ & $\begin{array}{l}\text { Volume } \\
\text { made } \\
\text { Up }\end{array}$ & $\begin{array}{l}\text { Concentration } \\
\text { ppm }\end{array}$ \\
\hline & \multicolumn{4}{|l|}{ High Range } \\
\hline 1 & $1000 \mu \mathrm{g} / \mathrm{Kg}$ & 10 & \multirow[t]{6}{*}{100} & 100 \\
\hline 2 & \multirow{5}{*}{$100 \mathrm{ppm}$} & 1 & & 1 \\
\hline 3 & & 2 & & 2 \\
\hline 4 & & 5 & & 5 \\
\hline 5 & & 7 & & 7 \\
\hline \multirow[t]{2}{*}{6} & & 10 & & 10 \\
\hline & \multicolumn{4}{|c|}{ Low range ppb \& Medium range } \\
\hline 7 & \multirow[t]{4}{*}{$1 \mathrm{ppm}$} & 0.5 & \multirow[t]{7}{*}{100} & 5 \\
\hline 8 & & 1 & & 10 \\
\hline 9 & & 2 & & 20 \\
\hline 10 & & 3 & & 30 \\
\hline 11 & \multirow[t]{3}{*}{$5 \mathrm{ppm}$} & 1 & & 50 \\
\hline 12 & & 2 & & 100 \\
\hline 13 & & 4 & & 200 \\
\hline
\end{tabular}

\section{DMA measurements}

The DMA-80 Direct mercury analyzer (Milestone, Inc.) Model write the steps of the measurement only.

Sudanese Ministry of Oil and Gas, Petroleum Laboratories Research and studies was used to determine the mercury concentration .

Other instruments based on these principles may also be appropriate. Analytical balance, (Sartorius 00578) maximum capacity $220 \mathrm{~g}$. or equiv. Compressor for oxygen high purity supplier glass pipettes, bulb, 1, 2, 5 \& $10 \mathrm{ml}$, Volumetric flasks class A or B (50 and $100 \mathrm{ml})$. 


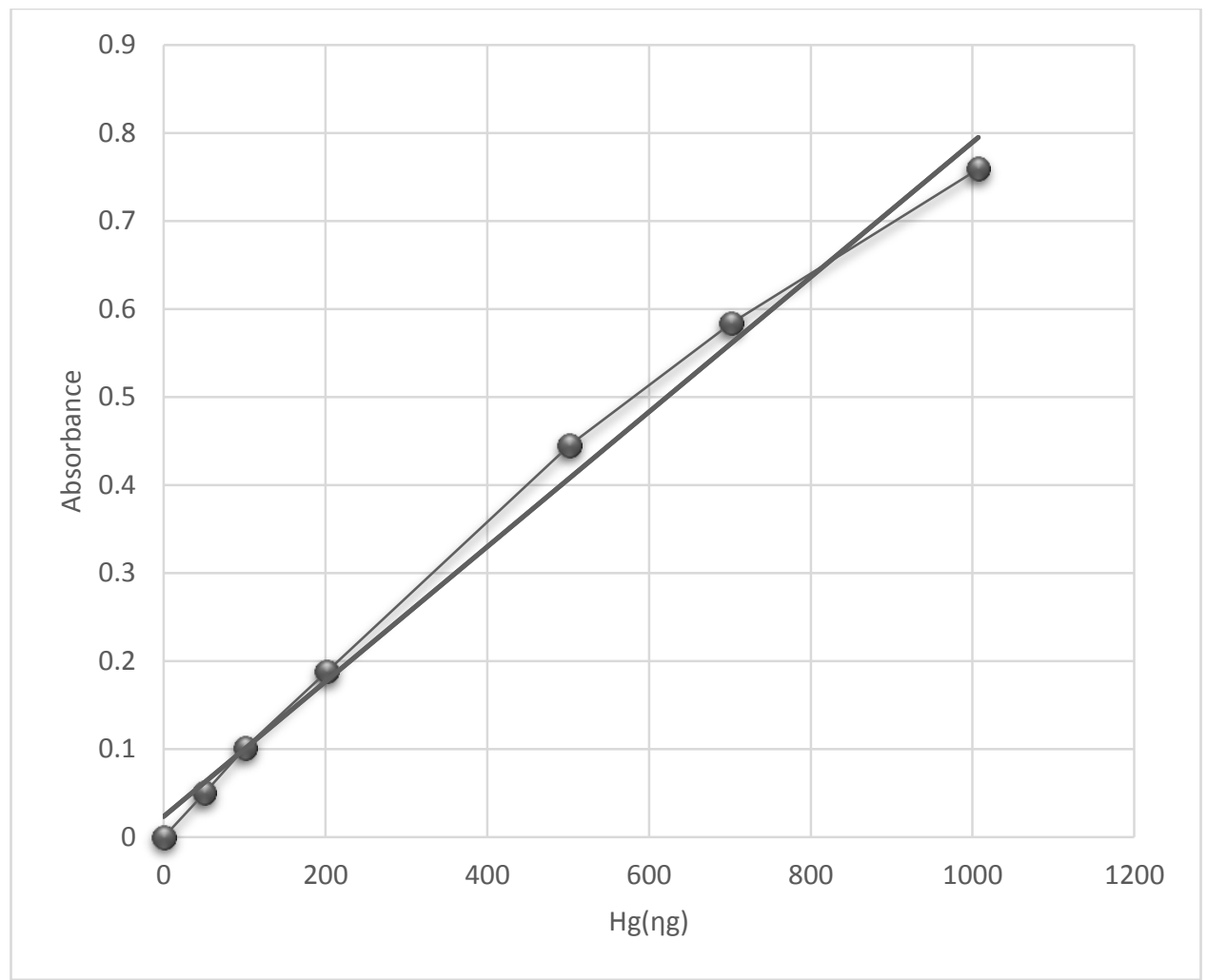

Fig 1:-Calibration curve of DMA-80

\section{Results and Discussion:-}

Mercury concentrations in human hair samples:

Direct Mercury analyzer (DMA) was used to determine mercury concentration in human hair samples. The average concentrations of mercury along with standard deviation (SD) values, the maximum value of concentration and the minimum value of concentration are listed in Table 1. As shown in Table 2, there was a striking difference in the concentration of mercury in different locations. The average concentration of $\mathrm{Hg}$ were 9.4 and $24 \mathrm{ppb}$ in location $1 \&$ 2 respectively, with standard division 8 and $15 \mathrm{ppb}$ for locations $1 \& 2$ respectively. Compared with the average values, the SD values are relatively large, but the SD values merely reflect the distribution of mercury concentrations due to the biological variability of each individual. The results show that the concentration of $\mathrm{Hg}$ in location one ranges between 1 and $41 \mathrm{ppb}$ with an average value of $9.4 \mathrm{ppb}$ and standard division value of $8 \mathrm{ppb}$. The concentration of $\mathrm{Hg}$ in location 2 ranges between 1 and $402 \mathrm{ppb}$ with an average value of $24 \mathrm{ppb}$ and standard division value of $15 \mathrm{ppb}$. According to the average concentration values in the studied locations. Mercury concentration values were higher in location 2 compared to its values in location 1 . The difference in mercury concentration between different locations in this study, suggests that increase of $\mathrm{Hg}$ caused by combustion step in mining process which it take place in location 2.

Table 2:-Summary of statistical data for mercury concentrations (ppb) in hair samples in different locations

\begin{tabular}{|l|l|l|l|l|}
\hline Hg & Mean & SD & Max & Min \\
\hline Loc1 & 9.4 & 8 & 41 & 1 \\
\hline Loc2 & 24 & 15 & 402 & 1 \\
\hline
\end{tabular}




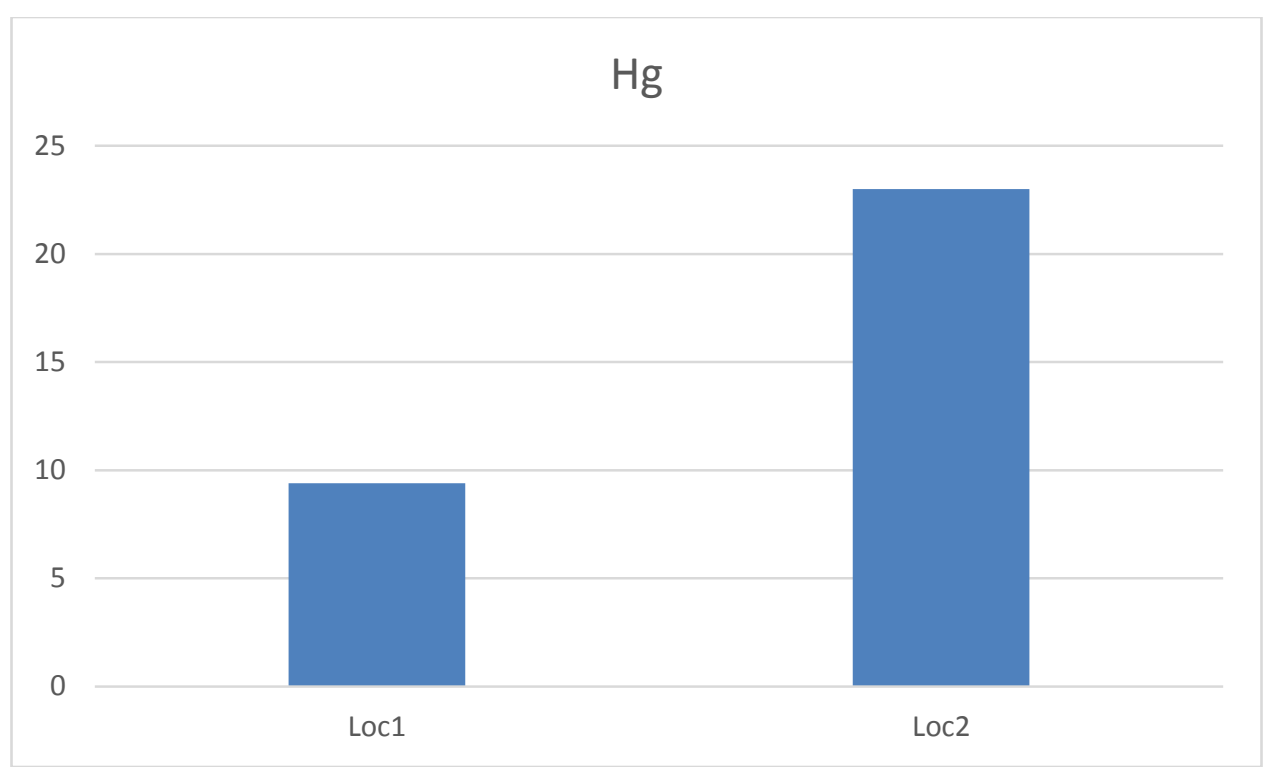

Fig 2:-Mean elemental concentration of $\mathrm{Hg}$ in different locations

\section{Conclusion and Recommendations:-}

Mercury in hair samples were analyzed in the present study. The results showed that levels of mercury in location 2 are higher than its concentration in location 1 . Recommendation of this study are awareness of dangers of mercury especially for miners, flow an occupational safety rules in traditional mining. Further studies are needed to expand samples numbers and new locations will be under study.

\section{Reference:-}

1. Robin A. Bernhoft "Mercury Toxicity and Treatment: A Review of the Literature" Journal of Environmental and Public Health Article I D 460508, 10 pages, (2012).

2. Francesco Domanico, Giovanni Forte Costanza, Majorani, Oreste Senofonte, Francesco Petrucci, Vincenzo Pezzi, Alessandro Alimonti "Determination of mercury in hair: comparison between gold amalgamation-atomic absorption spectrometry and mass spectrometry" Journal of Trace Elements in Medicine and Biology, (2016).

3. I. M. Fuentes and R. G. Martínez "Mercury Concentration i n Hair Due to Environment on Two Populations in Mexico" () Springer International Publishing AG (2018)

4. P. Li , X.B. Feng *, G.L. Qiu, L.H. Shang, Z.G. Li "Mercury pollution in Asia: A review of the contaminate d sites" Journal of Hazardous Materials 168 (2009) 591-601.

5. Amir M. Ashrafi, Zuzana Koudelkova, Eliska Sedlackova, Lukas Richtera, and Vojtech Adam "ReviewElectrochemical Sensors and Biosensors for Determination of Mercury Ions" Journal of the Electrochemical Society, 165 (16) B824-B834 (2018).

6. Echegaray, R., and M. Gómez. "Determinación de mercurio en cabello como expresión de la exposición a mercurio." Bol. Lima 6.31 (1984): 92.

7. Teresa Lech, and Wioletta Turek "Application of TDA AAS to Direct Mercury Determination in Postmortem Material in Forensic Toxicology Examinations" Journal of Analytical Toxicology, 1-7, (2019). 